\title{
Research on the Pricing of Electricity Swing Options
}

\author{
Mingzhu Wu \\ School of Management, Shanghai University, Shanghai, China
}

Keywords: swing options; electricity market; complementary model; M-matrix

\begin{abstract}
Since the electric power cannot be stored for long time, the spot prices of electricity are extremely volatile. In order to control risks, it is necessary to introduce financial derivatives into electricity markets. This paper mainly studies the pricing of electricity swing options, which are widely applied in financial markets for electricity. Through finite difference and discretization of transaction time and price, the issue of swing option pricing is transformed to a linear complementary problem. At the same time, the optimization model is established by combining optimal behaviors of swing option buyers. Finally, through the actual data of electricity futures, above model and algorithm are used to simulate the pricing of swing options.
\end{abstract}

\section{Introduction}

Swing options are also known as take-and-pay options. The contract lets option holders buy underlying assets repeatedly according to the bid price in the holding period. But the maximum and minimum quantities of underlying assets an option holder can buy at one time are predetermined, so as the total assets bought by the holder during the whole holding period. During the exercise period, option holders can change (swing) the pace of purchases, but generally speaking, the number of changes is also limited.

Thanks to the flexible delivery, electricity swing options are now considered as the most convenient and important financial derivatives among various financial instruments in the electricity market. The sellers of electricity swing options are mostly large electricity plants; the buyers are generally public electricity distributors. They set the retail price of electric power, and face the risks of electrical load and spot price. It is necessary for them to buy swing options to hedge risks.

Option holders can decide the delivery quantity and exercise time according to optimal strategy, while sellers have the right to set upper and lower bounds of delivery price and quantity. Swing options pricing is a complex issue.

Literature on swing option pricing without regard to underlying assets includes Eydeland and Geman (1998) as well as Davison and Anderson (2003) [1-2]. According to the no arbitrage principle, the value of swing options is calculated for option holders, so as to achieve swing option pricing. Thompson (1995), as well as Carmona and Touza (2006) take the perspective of buyers, and consider swing options as American options which can be exercised for many times [3-4]. Lari (2001) and Jaillet (2004) take the issue of swing options pricing as buyers' stochastic dynamic programming problem, in which the price changes of underlying commodities can be processed through binary tree or forest tree methods [5-6]. Longstaff and Schwartz (2001), as well as Ibanez (2004) assume that the price of underlying commodity is amenable to the mean reversion model [7-8]. Based on that, the Least Squares Monte Carlo simulation method is employed to solve the problem of swing option pricing.

As for the spot pricing of electricity swing options, Haarbrucker and Kuhn (2009) take it as the multistage stochastic optimization problem of buyers and sellers of swing options. The optimal value is equivalent to the expected economic benefits electricity swing options can bring for holders, in which the price of electricity can be processed through binary tree method [9]. At the same year, Broussev and Pflug (2009) analyze the optimal decisions of sellers and buyers, and draw the penalty function in case of option holders break the contract (the quantity or number of purchase is out of scope) [10]. Considering that the decision of both sides can affect each other's' optimal decision, 
Broussev and Pflug (2014) regard the pricing of electricity swing options as a bi-level programming problem, in which the seller of electricity swing options is the top decision maker who formulates different objective functions according to market situation [11].

However, electricity spot price fluctuates greatly. It is difficult to predict the price accurately. Direct delivery occupies only a small part of electricity market. Large electricity producers and dealers tend to control the risk of spot price through financial derivatives; well-developed electricity futures are used most frequently.

Based on above reasons, this paper takes a different perspective from previous studies. The electricity futures, rather than the swing option of spot electricity, are considered as the underlying assets. According to the requirements of both sides, buyers and sellers can sign the electricity delivery contract on the grounds of appropriate delivery mode. This method is suitable for practical application, and makes up for the deficiency of non-storage electric power. The non arbitrage principle can be used to price it. Combined with the optimal behavior of buyers, an optimization model can be set to achieve the pricing of electricity swing options.

\section{Option Pricing Model for Electricity Swing Futures}

\subsection{Analysis on the pricing of swing options on electricity futures}

The contract of electricity swing options allow multiple times of exercise in the contract period, but the time interval is predetermined. For the sake of simplicity, we consider each exercise happens in an equally divided time interval. The contract duration is equally divided into $T$ stages, i means at which stage, $t_{i}$ means at which time node, in which $i \in\{1,2, \cdots, T\}$. In each stage, the buyer of swing option must exercise for one time, but he has the right to decide when to exercise and the delivery quantity.

In order to better analyze the issue of electricity swing option pricing, the quantity of electric futures delivered at one time is set as one unit. Researchers start from the time period of the last $\mathrm{T}$ stage, [ ${ }_{T-1},{ }_{T}$ ]. If only this period of time is considered, the buyer of the swing option can decide to deliver a unit of electricity futures at any time in this period. At this point, the price of the electricity swing option at the time of $t_{T-1}$ is equivalent to the price of a futures contract which can be delivered ahead of time. Since the subject matter is electric electricity futures, the principle of no arbitrage can be used to achieve pricing. In the risk-neutral world, when only the time period of $\left[{ }_{T-1}, t_{T}\right]$ is considered, the Black-Scholes-Merton partial differential equation of the swing futures option at the time of $t_{T-1}$ is:

$$
\frac{\partial V}{\partial t}+\frac{1}{2} \frac{\partial^{2} V}{\partial F^{2}} \sigma^{2} F^{2}=r V
$$

At that time, the pricing of swing options is similar to the pricing of American options. Based on Black-Scholes-Merton partial differential equations, the pricing of American option is achieved. In this paper, finite difference processing method is used to solve the problem of option pricing. Finite difference method can convert Black-Scholes-Merton partial differential equations into differential equations which are relatively easy to solve, and transform the pricing issue into a complementary problem according to the characteristics of American options. Then the algorithm of complementary problem can be used to achieve the solution.

In reference to [12], the pricing problem of formula (1) can be written as following partial differential inequalities: 


$$
\left\{\begin{array}{l}
\frac{\partial V}{\partial t}+\frac{1}{2} \frac{\partial^{2} V}{\partial F^{2}} \sigma^{2} F^{2}-r V \leq 0 \\
V(F, t)-\Lambda(F, t) \geq 0 \\
\left(\frac{\partial V}{\partial t}+\frac{1}{2} \frac{\partial^{2} V}{\partial F^{2}} \sigma^{2} F^{2}-r V\right)(V(F, t)-\Lambda(F, t))=0
\end{array}\right.
$$

Through difference approximation, the left end of the partial differential inequality can be approximated as:

$$
\begin{aligned}
& -\frac{\partial V}{\partial t}-\frac{1}{2} \frac{\partial^{2} V}{\partial F^{2}} \sigma^{2} F^{2}+r V \approx V(F+\delta F, t)\left(-\frac{1}{2} \sigma^{2} F^{2} \theta \frac{1}{(\delta F)^{2}}\right) \\
& +V(F, t)\left(\frac{1}{\delta t}+r+\sigma^{2} F^{2} \theta \frac{1}{(\delta F)^{2}}\right) \\
& +V(F-\delta F, t)\left(-\frac{1}{2} \sigma^{2} F^{2} \theta \frac{1}{(\delta F)^{2}}\right) \\
& +V(F+\delta F, t+\delta t)\left(-\frac{1}{2} \sigma^{2} F^{2}(1-\theta) \frac{1}{(\delta F)^{2}}\right) \\
& +V(F, t+\delta t)(F+\delta F, t+\delta t)\left(\frac{1}{\delta t}+\sigma^{2} F^{2}(1-\theta) \frac{1}{(\partial F)^{2}}\right) \\
& +V(F-\partial F, t+\partial t)(F+\partial F, t+\partial t)\left(-\frac{1}{2} \sigma^{2} F^{2}(1-\theta) \frac{1}{(\delta F)^{2}}\right)
\end{aligned}
$$

According to above finite difference approximation, the finite dimensional linear complementary problem can be transformed into a set of finite dimensional linear complementary problems:

$$
0 \leq\left(V_{T-1}^{l}-\Lambda\right) \perp\left(M V_{T-1}^{l}+M^{\prime} V_{T-1}^{l+1}\right) \geq 0, \quad l=L-1, L-2, \cdots, 1,0
$$

In which $\perp$ means that two vectors are orthogonal, and the inner product is zero. For example, $x \perp y$ means $x^{T} y=0, V_{T-1}^{l}$ and $\Lambda$ are the following $\mathrm{N}$ dimensional vectors.

$$
V_{T-1}^{l} \equiv\left(\begin{array}{c}
V_{T-1,1}^{1} \\
\cdots \\
V_{T-1, N}^{l}
\end{array}\right) \quad \Lambda_{T-1}^{l} \equiv\left(\begin{array}{c}
\Lambda_{1} \\
\cdots \\
\Lambda_{N}
\end{array}\right)
$$

Where $\mathrm{M}$ is a matrix of $N \times N$ as following:

$$
M=\left(\begin{array}{ccccccc}
b_{1} & a_{1} & 0 & 0 & 0 & \cdots & 0 \\
a_{2} & b_{2} & a_{2} & 0 & 0 & \cdots & 0 \\
0 & a_{3} & b_{3} & a_{3} & 0 & \cdots & 0 \\
\vdots & \vdots & & & & & \vdots \\
0 & 0 & \cdots & 0 & a_{N-1} & b_{N-1} & a_{N-1} \\
0 & 0 & \cdots & 0 & 0 & a_{N} & b_{N}
\end{array}\right)
$$

Elements in $\mathrm{M}$ are:

$$
\begin{gathered}
a_{n}=-\frac{1}{2} \sigma^{2} n^{2} \theta, \quad n=1, \cdots, N \\
b_{n}=r+\frac{1}{\delta t}+\sigma^{2} n^{2} \theta, \quad n=1, \cdots, N
\end{gathered}
$$

$\mathrm{M}^{\prime}$ is a matrix shaped like $\mathrm{M}$, and the elements in the matrix are as following: 


$$
\begin{aligned}
& a_{n}{ }^{\prime}=-\frac{1}{2} \sigma^{2} n^{2}(1-\theta), \quad n=1, \cdots, N \\
& b_{n}{ }^{\prime}=-\frac{1}{\delta t}+\sigma^{2} n^{2}(1-\theta) \quad, \quad n=1, \cdots, N
\end{aligned}
$$

If the holder exercises at the deadline $t_{T}$, the price of swing option at the time of $t_{T-1}$ is equal to the value of income function, that is $V_{T-1}^{L}=\Lambda$. When the electricity swing option buyer set the delivery price as K, $\Lambda$ can be regarded as given. At that time, the linear complementary problem (3) can be solved by reversing the discrete time points $l=L-1, L-2, \cdots, 1,0 . V_{T-1}^{0}$ can also be calculated.

Now, the actual duration of electricity swing options is $\left[0,{ }^{t_{T}}\right]$; the total duration is divided into $\mathrm{T}$ stages. In each stage, the price of swing options in the time period of $\left[t_{i}, t_{i+1}\right], i \in\{0,1, \cdots, T-1\}$ is considered. In this paper, the finite difference method is used to transform the Black-Scholes-Merton partial differential equation to linear complementary constraint, which can be directly applied to solve the problem of price of multi-stage derivatives which can be exercised in advance. The following equation is established:

$$
V_{i-1}^{L}=V_{i}^{0}, \quad i=1,2, \cdots, T
$$

$V_{i}^{0}$ in each stage is the $V_{i-1}^{L}$ in previous stage. $V_{i-1}^{0}$ in previous stage can be obtained by inversion. Therefore, we can get $V_{0}^{0}$ after inversion step by step. Assuming that the quantity of deliveries is one unit, $V_{0}^{0}$ is the price of electricity swing option at the initial time.

\subsection{Behaviors of option buyers}

The valuation of any contract should include the optimal exercise strategy of option owners. Both hedgers and speculators aim to maximize their profits. Since the prices of electricity futures are stochastic, the simplest objective function of the contract buyer is to maximize the expected return.

The buyer of swing option can set the delivery price of each unit of electricity futures as K when signing the contract, and has the right to decide the delivery quantity at each stage in the future. If the amount of electricity futures expected to be delivered at each stage is $z_{i}, i \in\{1,2,3, \cdots, T\}$, then $Z_{i}$ is the amount of delivery within the time period of $\left[t_{i-1}, t_{i}\right]$. In the real financial market, the quantities of deliveries must be decided in advance for a period of time (usually one day). Thus, the buyer needs to decide the delivery quantity at next stage in advance.

Usually, the contract states the upper and lower bounds of delivery quantity at each stage, so as the total amount of delivery. The constraints associated with the number of deliveries can be expressed as

$$
\begin{array}{r}
\underline{e_{i} \leq z_{i} \leq \overline{e_{i}} \quad \forall i \in\{1,2,3, \cdots, T\}} \\
\underline{E} \leq \sum_{i=1}^{T} z_{i} \leq \bar{E}
\end{array}
$$

The formula (5) represents the upper and lower bounds of ${ }^{Z_{i}}$, the number of deliveries in the i stage, while formula (6) represents the upper and lower bounds of the total delivery quantity in the contract duration. Combining with the objective functions of electricity swing option buyers, which is to maximize the expected earnings, and supposing that the seller of swing option has set the delivery price as $\mathrm{K}$, and the contract is a call option, the optimization problem of buyers can be 
described as:

$$
\begin{aligned}
\max _{z_{i}} & \sum_{i=1}^{T} e^{-r t_{i-1}} z_{i}\left(V_{t_{i-1}}^{0}-K\right) \\
\text { s.t. } & \underline{e_{i}} \leq z_{i} \leq \overline{e_{i}} \quad \forall i \in\{1,2,3, \cdots, T\} \\
& \underline{E} \leq \sum_{i=1}^{T-} z_{i} \leq \bar{E}
\end{aligned}
$$

\section{Model Calculation}

Solution of linear complementary problem. Firstly, the properties of complementary problem of electricity swing option pricing are analyzed. In order to explain the issue more intuitively, $x=V_{T-1}^{l}-\Lambda$ and $q=M x+M \Lambda+M^{\prime} V_{T-1}^{l+1}$ are transformed. It should be noticed that since the reverse deduction method is used, $V_{T-1}^{l}$ can be regarded as given in the time period of $\left[t_{T-1}^{l}, t_{T-1}^{l+1}\right]$, and the problem of (3) can be simplified as following:

$$
x \geq 0, \quad M x+q \geq 0, \quad x^{T}(M x+q)=0
$$

In view of above problems, the existence and uniqueness of the solution are proved, and the following lemma is proposed in reference [13].

Lemma 3.1: if a square matrix $\mathrm{A}$ is a strictly diagonally dominant matrix, and all diagonal elements in $\mathrm{A}$ are positive, then $\mathrm{A}$ is a $\mathrm{P}$ matrix.

The matrix mentioned above $A=\left(a_{i j}\right)$ is a strictly diagonally dominant matrix, which is equivalent to following formula:

$$
\left|a_{i i}\right|>\sum_{j \neq i}\left|a_{i j}\right|, \quad i=1,2, \cdots, n
$$

If a square matrix is a P matrix, all its principal minors are positive. According to reference [14], following conclusions on P matrix are established.

Proposition 3.1: if a square matrix $A \in \mathfrak{R}^{n \times n}$ is a $\mathrm{P}$ matrix, then the linear complementary problem has a unique solution for any $q \in \mathfrak{R}^{n}$.

$$
0 \leq x \perp A x+q \geq 0
$$

Theorem 3.1: the matrix $M$ in the complementary problem transformed from the pricing model of electricity swing options is a P matrix, and the complementary problem has a unique solution.

Proof: first, it is obvious that the diagonal elements of matrix $\mathrm{M}$ are positive. According to Lemma (3.1), in order to prove that the matrix $\mathrm{M}$ is a $\mathrm{P}$ matrix, it is necessary to prove that the matrix $\mathrm{M}$ is a strictly diagonally-dominant matrix. Notice that all $b_{n}, n=1 \cdots, N$ are positive, and all $a_{n}, n=1, \cdots, N$ are negative, then:

$$
\begin{aligned}
& \left|b_{1}\right|-\left|a_{1}\right|=r+\frac{1}{\delta t}+\sigma^{2} \theta-\frac{1}{2} \sigma^{2} \theta=r+\frac{1}{\delta t}+\frac{1}{2} \sigma^{2} \theta \\
& \left|b_{n}\right|-2\left|a_{n}\right|=r+\frac{1}{\delta t}+\sigma^{2} n^{2} \theta-\sigma^{2} n^{2} \theta=r+\frac{1}{\delta t}, n=2, \cdots, N-1 \\
& \left|b_{N}\right|-\left|a_{N}\right|=r+\frac{1}{\delta t}+\sigma^{2} N^{2} \theta-\frac{1}{2} \sigma^{2} N^{2} \theta=r+\frac{1}{\delta t}+\frac{1}{2} \sigma^{2} N^{2} \theta
\end{aligned}
$$

Obviously, the values of above equations are greater than zero. So the matrix $\mathrm{M}$ is a $\mathrm{P}$ matrix. According to Lemma (3.1), there is a unique solution to the complementary problem transformed from the pricing model of electricity swing options in this paper.

The coefficient matrix $\mathrm{M}$ has a lot of good properties, and the size of the linear complementary 
problem is becoming larger and larger as the difference step size gets smaller and smaller. So in this paper, though interior point method and smoothing Newton method have good convergence and accuracy, they are not applicable to solve the linear complementary problem due to high computational cost. The solution in this paper is similar to fixed point algorithm at the beginning. The linear complementary problem is transformed to a set of large-scale linear equations with fixed points first. Then the over relaxation iteration method is used to solve to large-scale linear equations set.

\section{Numerical Experiment}

Based on actual data about electricity futures provided by Chicago Mercantile Exchange, the model and algorithm provided in this paper are used to price electricity swing options. A valid contract on electricity futures is selected from CME official website. ( The underlying futures are electricity futures which has been traded in New York Electricity Exchange for a month; each unit of future permits the delivery of 5 megawatt hour.) The duration is 30 days ( $t_{T}=30$ days). One time of delivery is permitted every day (the duration can be divided into 30 stages, $T=30$ ). Assuming that the amount of delivery at one time lies between 1 and 3 units of electricity futures, the total delivery amount is 40 to 60 units of electricity swing options.

Next, the model and algorithm proposed in this paper are used to achieve the pricing of electricity swing option. First of all, when building the model, parameters $r, \sigma, \theta$ and $\mathrm{r}$ should be set as risk-free interest rates. The yields on 10 -year Treasury bonds, $r \approx 2.54 \%$, is generally chosen.

$\sigma$ represents the volatility of the random process of electricity futures price. Through historical data provided by CME and seasonal characteristics, assuming that the trading month of electricity swing options is the whole April (30 days), through data a few years ago to estimate the volatility of electricity futures price of electricity swing options, the volatility is estimated as $\sigma_{1} \approx 32.55 \%$.

Secondly, the difference parameter $\theta$ is selected. The Crank-Nicolson difference method is used according to reference [14], namely $\theta=1 / 2$

Now consider the price of electricity futures and time difference step. From historical data it can be found that electricity futures price is no less than $\$ 15$ and no more than $\$ 30$ per unit in April. The difference step of electricity futures price is selected as $N=100$; the length of difference step is selected as $\delta F=0.15$; the time difference step as $L=24$. The length of difference step is selected as $\delta F=0.15$, means to divide one day into 24 hours.

With the assumption of above parameters, the elements of $a_{n}$ and $b_{n}$ in matrix $\mathrm{M}$ can be obtained:

$$
\begin{aligned}
& a_{n}=-\frac{1}{4} 0.3255^{2} n^{2}, \quad n=1, \cdots, N \\
& b_{n}=0.0254+24+\frac{1}{2} \sigma^{2} 0.3255^{2} n^{2}, \quad n=1, \cdots, N
\end{aligned}
$$

Similarly, we can get the elements of $a_{n}{ }^{\prime}$ and $b_{n}{ }^{\prime}$ in matrix $\mathrm{M}^{\prime}$ :

$$
\begin{aligned}
& a_{n}{ }^{\prime}=-\frac{1}{4} 0.3255^{2} n^{2}, \quad n=1, \cdots, N \\
& b_{n}{ }^{\prime}=-24+\frac{1}{2} 0.3255^{2} n^{2} \quad, \quad n=1, \cdots, N
\end{aligned}
$$

Assuming that the optimal delivery price $K=22$, and substituting it into problem (2.3), the solution of $V_{0}^{0}$ is a matrix with 100 rows and 1 column. From CME data it can be known that the initial price of electricity futures is $\$ 19.73$, corresponding to the interval of electricity futures price 
in $V_{0}^{0}$. The price of electricity swing option per delivery unit is calculated as approximate \$23.7. Then considering buyers' optimal purchase amount at each stage, the electricity swing option price is finally obtained as $\$ 2004$.

Besides, this paper also selects different step lengths of time difference without regard to seasonal influence. According to data of the first few months, the volatility rate is gotten as $\sigma_{2} \approx 43.74 \%$. The prices of electricity swing options are calculated as follows.

\begin{tabular}{|c|c|c|c|c|}
\hline Time difference & 1 day & 4 hours & 1 hour & 30 minutes \\
\hline$\sigma_{1}$ & 2328.3692 & 2396.8280 & 2004.1351 & 1458.8847 \\
\hline$\sigma_{2}$ & 3344.8208 & 3171.2810 & 2441.8448 & 1578.7603 \\
\hline
\end{tabular}

It can be found that with the decrease of difference step length and the increase of the difference step number, the prices of electricity swing option are constantly revised and reduced. Under the same length, electricity swing option price increase with the rising volatility: it accords with the actual situation. So the overall experiment prices are higher because of the high volatility of electricity futures price. It can be inferred in summer and winter (volatility are higher), the electricity swing option prices should be even higher, which is also consistent with the reality.

\section{Conclusion and Outlook}

In this paper, the pricing of electricity swing options is achieved with electricity futures considered as the underlying asset. Through finite difference and discretization of transaction time and price, the issue of swing option pricing is transformed into a linear complementary problem. Furthermore, combined with optimal behaviors of buyers, the pricing of electricity swing option can be more accurate. It can be found that optimal decisions of buyers and sellers can affect each other, so a bi-level programming model is established to price the electricity swing options. At the same time, in reality, buyers do not need to strictly control the purchase amount at each stage in accordance with sellers' permission. Purchase amount less than lower bound or more than upper bound is acceptable, but the buyer need to pay the penalty for breach of contract. So buyers' optimal behavior can be considered as mathematical programming problems with penalty function, rather than trading restriction in purchase amount.

\section{References}

[1] A. Eydeland, H. Geman, Some fundamentals of electricity derivatives, J. Automatica. 3 (1998).

[2] M. Davison, L. Anderson, Approximate recursive valuation of electricity swing options, J. European Journal of Operational Research. 17 (2003).

[3] A. Thompson, Valuation of path-dependent contingent claims with multiple exercise decisions over time: The case of take-or-pay, J. Journal of Financial and Quantitative Analysis. 30 (1995).

[4] R. Carmona, N. Touzi, Optimal multiple stopping and valuation of swing options, J. Mathematical Finance. 18 (2008).

[5] A. Lari-Lavasanni, M. Simchi, A discrete valuation of swing options, J. Canadian Applied Mathematics Quarterly. 9 (2001).

[6] P. Jaillet, Valuation of commodity based swing options, J. Management Science. 50 (2004).

[7] F. Longsta, E. Schwartz, Valuing American options by simulation: A simple least squares approach, J. The Review of Financial Studies. 14(2001).

[8] A. Ibanez, Valuation by simulation of contingent claims with multiple exercise opportunities, J. Mathematical Finance. 14(2004).

[9] G. Haarbrucker, D. Kuhn, Valuation of electricity swing options by multistage stochastic programming, J. Automatica. 45(2009). 
[10]C. Georg, N. Broussev, Electricity swing options: Behavioral models and pricing, J. European Journal of Operational Research. 19(2009).

[11]M. Raimund, C. Georg, Electricity swing option pricing by stochastic bilevel optimization: A survey and new approaches, J. European Journal of Operational Research. 27(2014).

[12]K. Hamatani, M. Fukushima, Pricing american options with uncertain volatility through stochastic linear complementary, J. Computational Optimization and Applications. 50(2011).

[13]O. Svensson, S. Vorobyov, A subexponential algorithm for a subclass of P-matrix generalized linear complementary problems, J. Technical Report. 16(2005).

[14]J. Zhao, R. M. Corless, Compact finite dierence method for American option pricing, J. Journal of Computational and Applied Mathematics. 206(2007). 